\title{
Kapitel 2 - Typen sozialer Abwehrhandlungen
}

Vor dem Hintergrund der Darstellung und Synthese der theoretischen Befunde sollen nun verschiedene Formen von Abwehrhandlungen vorgestellt werden. Hierbei wird deutlich werden, dass bezüglich der Analyse von deren Ausgestaltung und Wirksamkeit stets die soziale Position des oder der handelnden Akteur*in sowie der soziale Kontext und die soziale Funktion der Handlung berücksichtigt werden muss.

In Kapitel 2.1 werden unter der Kategorie Modifikation und unter Kapitel 2.2 unter der Kategorie Kommunikationsvermeidung Formen von Abwehrhandlungen diskutiert, die gemein haben, dass sie darauf zielen, präventiv die Wahrscheinlichkeit herabzusetzen, dass eine antisemitische Äußerung oder ein (vergangenes) antisemitisches Ereignis als Problem adressiert wird. Erweisen sich solche Verhaltensweisen als erfolgreich, so entsteht überhaupt nicht erst der Bedarf, einen manifesten antisemitischen Konflikt neutralisieren zu müssen, weil ein objektiv bestehender antisemitischer Konflikt latent gehalten werden kann. $\mathrm{Zu}$ den, hinsichtlich der Erweiterung des öffentlich Sagbaren, >innovativen Modifikationen des Antisemitismus gehören insbesondere die Formulierung eines >demokratischen Antisemitismus, die Camouflage von Antisemitismus, sowie die Umwegkommunikation, Disclaimer und Selbstkorrekturen. Unter Kommunikationsvermeidung werden demgegenüber insbesondere das (beredte) Schweigen und die Verwendung von Narrativen gefasst, die die Funktion erfüllen, das Thema Antisemitismus zu marginalisieren. Diese Verhaltensweisen setzen die Existenz öffentlicher Konflikte über Antisemitismus voraus und sind als Reaktion hierauf verstehbar, erweisen sich aber vor allem in solchen Situationen als wirkungsvoll, in denen die Wahrscheinlichkeit gering ist, dass jemand aktuell anwesend, handlungsfähig oder gewillt ist, Antisemitismus nachdrücklich zu kritisieren.

Alle anderen diskutierten Formen von Abwehrhandlungen setzen demgegenüber eine (öffentlichkeits-)wirksame Problematisierung von Antisemitis- 
mus voraus, der nicht einfach ausgewichen werden kann und zielen darauf, den desintegrativen Folgen von Antisemitismuskritik zu begegnen. So sind Formen der starken und der abgeschwächten Rechtfertigung (Kapitel 2.3) der antisemitischen und nicht-antisemitischen Aufrechnung von Antisemitismus (Kapitel 2.4) und die situationsspezifische oder generalisierte moralische Diskreditierung von Antisemitismuskritik (Kapitel 2.5) als offensive Reaktionen gegen anti-antisemitische Interventionen oder eine etablierte Erinnerungskultur zu verstehen. Dies bedeutet, dass sie sich sowohl am Status der Opfer von Antisemitismus als auch am Status der Antisemitismuskritiker*innen abarbeiten und hierbei mit der Konstruktion verschiedener Feindbilder einhergehen. Demgegenüber lassen sich die verschiedenen Formen der Bagatellisierung von Antisemitismus (Kapitel 2.6) und die Leugnung antisemitischer Phänomene (Kapitel 2.7) als defensive Formen der Abwehr beschreiben, die ohne die Konstruktion von Feinbildern einhergehen.

Das Kapitel zu den Formen der sozialen Abwehr wird zum einen mit einer Beschreibung von externalisierenden und extremisierenden Darstellungen des Antisemitismus abgeschlossen, die Abwehrhandlungen häufig begleiten (2.8). Zum anderen wird auf den sekundären Antisemitismus (2.9) eingegangen, der als komplexes soziales und psychologisches Abwehrphänomen beschrieben werden kann.

\subsection{Modifikation antisemitischer Äußerungen}

Wie bereits in der Rezeption Werner Bergmanns deutlich wurde, hat das zumindest teilweise erfolgreiche Abdrängen antisemitischer Kommunikation aus der Öffentlichkeit dazu geführt, dass Antisemitismus nach 1945 in seinen traditionellen Formen zum privaten Massenvorurteil geworden ist. Da aber »Bewußtsein zur Kommunikation drängt« (Heyder et al. 2005: 156, vgl. auch Beyer/Krumpal 2010: 685), kann auch versucht werden sich antisemitisch auf eine Weise zu äußern, die die Wahrscheinlichkeit anti-antisemitischer Interventionen herabsetzt. Entsprechende Formen des Sprechens folgen hierbei Taguieff zufolge verschiedenen »Präsentations- und Repräsentationsstrategien [...], die es ihm erlauben, bestimmte Zulassungs- und Akzeptanzbedingungen zu erfüllen, die in der konkreten historischen Situation in der Öffentlichkeit gelten« (Taguieff 1991: 244).

Möglich wird dies zum einen durch Innovationen bezüglich des Modus der Konstruktion antisemitischer Stereotype und Vorurteile. So ist in der Antisemi- 
tismusforschung z.B. die Verwendung von antisemitischen Chiffren bekannt, wobei die Begriffe `Juden « und >Judentum< durch (vermeintlich oder tatsächlich) unbelastete Begriffe wie samerikanische Ostküsteく oder `Zionisten setzt werden. Hier bewirkt das Mittel der »Camouflage« (Holz 2001: 481), dass die Wahrscheinlichkeit von Widerspruch herabgesetzt wird. Eine modifizierte Form der Konstruktion antisemitischer Stereotype liegt auch dann vor, wenn explizit zwischen sguten 161) - d.h. z.B. zwischen >West-< und `Ostjuden<, jüdischen Kritiker*innen und vermeintlichen Parteigänger*innen Israels, sliberalen und silliberalen Jüd*innen, jüdischen Institutionen und jüdischen Menschen usw. - unterschieden wird, um antisemitische Vorurteile selektiv zu formulieren. Auch dies kann zur Akzeptanz von negativen Äußerungen über Jüd“innen führen, weil deutlich gemacht wird ist, dass man kein Pauschalurteil fällt, sondern nur ein Teilgruppe von Jüd*innen ablehnt. Zu einem sehr ähnlichen Phänomen kommt es bei der strategischen Bezugnahme auf Kronzeug*innen. Statt einer Distanzierung von Antisemitismus kommt es hierbei zu einer Indienstnahme von Autoritäten, die im Common-Sense-Bewusstsein unzweifelhaft keine Antisemit"innen sein können. So kann antisemitische Rede z.B. eingeführt werden, indem sich auf die »wahren oder fiktiven Äußerungen« meist jüdischer Personen bezogen wird, um zu berufen und Antisemitismus dadurch gerechtfertigt erscheinen zu lassen. Auf Kritik kann dann wiederum mit einer Distanzierung regiert werden: diese Äußerung sei ja nicht die eigene und man könne für sie also nicht zur Verantwortung gezogen werden.

In Anschluss an die These der Kommunikationslatenz kann weiterhin argumentiert werden, dass sich bestimmte negative Darstellungen Israels anbieten, um antisemitische Einstellungen folgenlos zu kommunizieren. Hierbei ist dann von Umwegkommunikation die Rede, die mit der Übertragung von antisemitischen Stereotypen auf den jüdischen Staat, seiner Dämonisierung, Delegitimierung und der Anwendung doppelter Standards operiert (vgl. Sharansky 2004: 3f.). In ihrer Analyse quantitativer Daten kommen Heyder und Kolleg*innen zu dem Ergebnis, dass sich neben dem israelbezogenen Antisemitismus auch der sekundäre Antisemitismus und die Infragestellung der Loyalität deutscher Jüd*innen zu Deutschland für die Umwegkommunikation anbieten (Heyder et al. 2005: 148).

Eine andere Form der Innovation besteht in der modifizierten Einführung antisemitischer Argumentationen und damit verbunden, der Selbstrepräsentation von antisemitisch Handelnden. So wurde der Begriff des demokratischen Antisemitismus dafür geprägt, um solche Formen des Antisemitismus 
zu bezeichnen, die »von der Demokratie her gegen die Juden« argumentieren, wobei sich Sprecher*innen als "human, aufgeschlossen und vorurteilsfrei« (Pollock 1955: 403) präsentieren. Im Feld des israelbezogenen Antisemitismus ist dies z.B. dann der Fall, wenn sich »im Namen der Menschenrechte, des Antirassismus und des Antikolonialismus« (Amadeu Antonio Stiftung 2017: 12) auf antisemitische Weise geäußert wird. Eine Variation dieses Schemas liegt vor, wenn sich zur Schuld und moralischen Verantwortung der Deutschen aufgrund der NS-Geschichte« bekannt wird, um genau hierüber die Notwendigkeit der eigenen (als antisemitisch kritisierbaren) Position zu begründen (vgl. Schwarz-Friesel 2012: 360). Dies geschieht z.B. bei »Was gesagt werden muss«, wenn Grass die Notwendigkeit seiner >Kritik« Israels aus dem Bewusstsein um die historische Schuld Deutschlands ableitet (siehe S. 140). Weiterhin besteht die Möglichkeit sich im Zuge antisemitischer Äußerungen als vermeintliche Tabubrecher*in zu inszenieren. Die Strategie des Tabubruchs setzt an der demokratischen Idee an, dass Tabus falsch wären und ihre Überwindung befreienden Charakter habe. Antisemitismus erscheint dann im »emanzipatorischen Gewand « (Weiß 2001: 255). Tabubrecher"innen stellen sich als Aufklärer*innen und Teil einer politischen Avantgarde dar, die gegen undemokratische Sprechverbote bzgl. >der Juden` oder Israel aufbegehre. ${ }^{1}$ Die Strategie des kalkulierten Tabubruchs, der auch von nachfolgenden Rechtfertigungen oder leichten Abmilderungen der getätigten Äußerung begleitet werden kann, ist insbesondere von der Neuen Rechten ideologisch entwickelt worden. Sie lässt sich in Antisemitismuskonflikten nach 1989 aber auch bei Linken, oder Menschen, die sich der demokratischen >Mitte $\mathrm{zu}$ ordnen, beobachten (vgl. Rensmann 2004: 344ff., Bleeker-Dohmen/Strasser 2009). ${ }^{2}$ Zuletzt kann die Einführung antisemitischer Stereotype mit Disclaimern, durch deren Verwendung eine explizite Distanzierung von Antisemitismus vollzogen wird, erneuert werden. Durch Disclaimer, die häufig ein »Jaaber-Äußerungsformat « (Holz 2007: 56, vgl. auch Schwarz-Friesel 2012: 365) annehmen, werden humanitäre Werte zunächst dem Schein nach und häufig phrasenhaft bestätigt, um dann eine antisemitische Äußerung anzuschließen tismuskritiker*innen in die eigene Verteidigung eingefügt (siehe Kapitel 2.5). reits im Titel an, durch den impliziert wird, dass etwas Wichtiges nicht ausgesprochen oder verschwiegen werde. 
»which is typically constructed through the prism of >realism, < truth, < or >common sense« (van Dijk 1992: 111; vgl. hierzu auch Chiang 2010: 278).

Eine dritte Möglichkeit der Innovation des Antisemitismus besteht demgegenüber in der Modifizierung des Modus seiner Äußerung, mit der die Wahrscheinlichkeit von Kritik herabgesetzt wird bzw. die mögliche Antisemitismuskritik ins Leere gelaufen lassen wird. So können etwa antisemitische Stereotype im raunenden Sprechen angedeutet werden, um die Rede dann abbrechen zu lassen oder abgewartet werden, wie auf Andeutungen reagiert wird, um dann den eigenen Antisemitismus offener zu artikulieren (vgl. Ranc 2016: 29f., Rensmann 2004: 179). Im Sinne von Habermas Theorem zu Kommunikationspathologien wird hierbei also aus strategischen Erwägungen heraus die Norm der Verständlichkeit verletzt, um einen offenen Konflikt $\mathrm{zu}$ vermeiden. Diesbezüglich ist in der Antisemitismusforschung auch vom »Kryptoantisemitismus« die Rede (vgl. Kistenmacher 2017: 206). Antisemit*innen verhalten sich hierbei in Gesprächssituationen auf taktische Art, indem sie den Möglichkeitsspielraum für antisemitische Kommunikation vermessen. Hiermit verwandt sind Phänomene, die Scott A. Hanson-Easey und Martha Augoustinos als sprachliche self-repair-Mechanismen bezeichnen. So könne sich z.B. im Stottern, unauffälligen Unterbrechungen der Rede und Formen von Selbstdementierungen anzeigen, "that the speaker is experiencing some trouble formulating his account as he expects some interactional trouble to arise from what he is saying " (Hanson-Easey/Augoustinos 2012: 43, vgl. auch Billig 1997: 148).

\subsection{Kommunikationsvermeidung}

Freilich können antisemitisch eingestellte Personen auch dazu tendieren, ihre Einstellungen nicht zu äußern, d.h. sie zu verschweigen (vgl. Bergmann 1991b: 506) oder sich einer Kontroverse über Antisemitismus zu entziehen (Byford 2013, V: 8). Im Sinne Max Webers ist Schweigen hierbei nicht als Nicht-, sondern als soziale Handlung - nämlich der des »Unterlassens« (vgl. Weber 1921: 1) - zu bezeichnen. In Anschluss an metapsychologische Überlegungen zur Genese von antisemitischen Vorstellungsgehalten (siehe S. 42) lässt sich diesbezüglich sagen, dass dieses Schweigen allerdings nicht deshalb gewählt wird, weil diesen Personen der gewaltvolle Charakter der eigenen Ansichten bewusst ist. Bewusst ist ihnen vor allem, dass die Kommunikation der eigenen Ansichten zu sozialen Konflikten und zu Prestigeverlust führen könnte. 
Schweigen als soziale Strategie wird allerdings nicht allein von Antisemit*innen, aufgrund möglicher anti-antisemitischer Interventionen des sozialen Umfelds, gewählt. Wie bereits in Anschluss an Sartre festgestellt (siehe S. 70), kann Schweigen auch von strategischer Bedeutung für nicht-antisemitische Personen sein, um so einem möglichen Konflikt mit Antisemit*innen aus dem Wege gehen zu können.

Drittens kann sich die Verhaltensweise des Schweigens auch als Reaktionsweise auf tatsächlich erfolgte Problematisierungen von Antisemitismus oder die Erinnerung an antisemitische Gewalt beziehen. So präsentiert etwa Gesine Schwan eine spezielle Form des »beredten Schweigens«, mit der nationalsozialistische Täter in Gerichtsverhandlungen auf Anklagen reagierten: Sie bekannten sich zu vergleichsweise kleinen Vergehen, um dadurch von den eigentlich größeren abzulenken. Dies wird von Schwan metapsychologisch und soziologisch als eine Art des unbewussten Kompromisses zwischen »dem Wunsch, sich einerseits als moralisches Subjekt anerkennen und präsentieren zu können und andererseits das Bekenntnis der eigentlichen Schuld abzuwehren« interpretiert (Schwan 1990: 106, Hervorhebung M.H.).

Weiterhin wird das Beschweigen der Shoah und des Antisemitismus in den unmittelbaren Nachkriegsjahren als eine der wichtigsten Umgangsweisen von Täter*innen und Nicht-Täter*innen mit dem Erbe des Nationalsozialismus im Allgemeinen und der Erinnerung des Judenmords im Speziellen betrachtet (vgl. Ahrendt 1986: 51; Horkheimer 1996: 739, Frei 2012: 29ff.). Sie stellte hierbei solange eine effektive Abwehrstrategie dar, wie ein unausgesprochener Gruppenkonsens darüber bestand, dass es für das eigene Ansehen, das Ansehen Nahestehender sowie das Ansehen Deutschlands dienlich wäre, sich weder mit vergangenem Antisemitismus noch mit der Gegenwart der Ideologie auseinanderzusetzen.

Die Chance der Thematisierung von vergangenem Antisemitismus wird aber auch dann herabgesetzt, wenn das Thema Antisemitismus und die Erinnerung an die Opfer antisemitischer oder rassistischer Verfolgung marginalisiert wird. Dies geschieht z.B., indem gruppenbezogene Narrative entwickelt werden, die die Unterdrückung von antisemitismus- und diskriminierungsrelevanten Informationen gewährleisten. Diese Unterdrückung wird Byford zufolge durch die Schaffung eines passenden »replacement myth « (Byford 2013, I: 18) erreicht. In selbstviktimisierenden Narrativen können sich Mitglieder der nicht-jüdischen Mehrheitsbevölkerung z.B. als das eigentliche Opfer der Geschichte darstellen (vgl. Möller 2006). Hierzu gehörte z.B. das vor einigen Jahren durch die NPD lancierte Sprechen über einen >Bom- 
benholocaust‘, der an der deutschen Bevölkerung durch die Alliierten verübt worden sei. ${ }^{3}$ Auf privater Ebene sind demgegenüber heroisierende Beschreibungen verbreitet, bei denen die Großeltern zu Rettern von Jüd*innen und politischen Dissident"innen stilisiert werden. Ihre Dominanz führt zu einer Marginalisierung bzw. einem kompletten Verschwinden von Erinnerungen an die Verstrickung der Großelterngeneration in das nationalsozialistische Verfolgungssystem (vgl. Welzer et al. 2002: 81ff.). Anders als in anderen Ländern (z.B. in Schweden, Serbien und Frankreich) ist allerdings eine die NS-Zeit betreffende heroisierende Gruppenerzählung zumindest in der deutschen Öffentlichkeit kaum anschlussfähig. Dies liegt Klaus Holz zufolge daran, dass der deutsche Nationalismus nach 1945 als "Nationalismus der >Mitte« nur fortgesetzt werden konnte, indem die Judenvernichtung zum »konstitutiven Bezugspunkt für das nationale Selbstverständnis" (Holz 2007: 51) gemacht wurde. Aus diesem Grunde beziehen sich selbstglorifizierende Erzählungen in Deutschland vor allem auf eine vermeintlich rundum gelungene Aufarbeitung der Vergangenheit, verbunden mitunter mit der These, dass Antisemitismus vollkommen verschwunden sei (vgl. Miller 2006: 124). Auch diese Narrative können als Form der Deckidentität in Diskussionen genutzt werden, um die Relevanz der Thematisierung von gegenwärtigem Antisemitismus herabzuspielen.

\subsection{Rechtfertigung}

Die meisten Formen öffentlicher antisemitischer Rede und Diskriminierung gehen mit dem Versuch ihrer Legitimation, d.h. einer Darstellung von Jüd*innen als Täter*innen und antisemitisch Handelnden als deren Opfer einher. Dies liegt daran, dass antisemitische Handlungen, ohne sie begleitende und legitimierende Argumentationen, stets dem Risiko ausgesetzt sind, als unbegründet zu erscheinen. Sie erfüllen damit den Zweck, verbale und körperliche Gewalt nicht als Gewalt, sondern als Ausdruck von rational begründeter Gegenwehr erscheinen zu lassen (vgl. Holz 2001: 160). Hierdurch wird zumindest indirekt eingestanden, dass antisemitische Handlungen, Narrative usw. begründungsbedürftig sind und sich nicht von selbst verstehen. Etabliert sich allerdings nennenswerter anti-antisemitischer Widerstand oder 
eine auf vergangenen Antisemitismus bezogene Erinnerungskultur, so kann der Bedarf entstehen, entsprechende Begründungen diskursiv nachzureichen oder zu erweitern, um antisemitische Handelnde gegen Kritik zu verteidigen, Antisemitisches sagbar zu halten bzw. die eigene antisemitische Einstellung $\mathrm{zu}$ rechtfertigen. In eben diesem Sinn sind Formen der Rechtfertigung auch als »blaming the victim« (vgl. Tileagă 2005: 618) oder als »primäre Form der Schuldabwehr « (Assmann 2011: 169) verstehbar. Entsprechende Argumentationen stellen die offensivste Strategie dar, mit anti-antisemitischem Widerspruch umzugehen. Sie ist allerdings auch mit hohen Risiken verbunden. Denn wird der antisemitische Konflikt, innerhalb dessen solche Strategien verwendet werden, verloren, so stehen die sich so äußernden nun als Gewalttäter*innen dar.

Bei der Rechtfertigung wird meist nichts, oder kaum etwas, vom kommunizierten Antisemitismus zurückgenommen. Stattdessen werden antisemitische Narrative weiterentwickelt, um Antisemitismuskritiker*innen dazu zu nötigen, eine neue, und eventuell schwächere anti-antisemitische Position zu vertreten. Dies kann z.B. bedeuten, einen abrupten Themenwechsel zu vollziehen und eine gänzlich andere Klasse vermeintlicher Verhaltensweisen von Jüd*innen oder Israelis zu benennen, die Kritik an der Gruppe notwendig mache. ${ }^{4}$ Allerdings können Rechtfertigungen auch als »Abschwächung« und »Abtönung « der vorurteiligen Position auftreten (vgl. Seu 2010: 441, van Dijk: 92). Dies ist insbesondere dann wahrscheinlich, wenn mit starken antiantisemitischen Gegenkräften gerechnet werden kann. Der gesellschaftliche Prototyp einer solchen Strategie bestand in einigen Antisemitismuskonflikten der Nachkriegszeit darin, einen vermeintlich moderaten Antisemitismus zu vertreten und sich hierbei vom Vernichtungsantisemitismus abzugrenzen. Dann war z.B. davon die Rede, dass es zwar ein Judenproblem gäbe, dass ihre Ermordung allerdings ein Fehler gewesen sei (vgl. Bergmann 1997: 119ff.). In Reaktion auf öffentliche Kritik wurde hierbei die eigene Position also nochmals paraphrasiert und im gleichen Zuge so weit abgeschwächt, dass damit die Wahrscheinlichkeit stieg, dass die sie nun als sozial akzeptabel rezipiert wurde.

In Anlehnung an Miller lässt sich sagen, dass hierbei durch die Einführung eines neuen, normativen Gesichtspunkts eine temporäre Patt-Situation mit Antisemitismuskritiker*innen angestrebt wird, um sich »gegenüber dem Gegner etwas Luft zu verschaffen« (Miller 2006: 52). 
Eine abgeschwächte Rechtfertigung von Antisemitismus kann drittens auch darin bestehen, zu versuchen, unter den Vorzeichen der Ablehnung von Antisemitismus im Allgemeinen, einer spezifischen Facette der Ideologie einen tieferen Sinn abzugewinnen und sie dadurch zu rationalisieren. So verweist Bergmann in Anschluss an Mario Kessler und Olaf Kistenmacher darauf, dass in manchen Strömungen der radikalen Linken wiederholt versucht wurde, dem politischen Antisemitismus eine "progressive KatalysatorFunktion « in der Auseinandersetzung mit dem Kapital oder Kapitalist*innen zuzuschreiben (vgl. Bergmann 2007: 19, vgl. auch Rürup 1975: 119). Gerechtfertigt werden hier also nicht antisemitische Inhalte, sondern ihre vermeintliche Wirkung. $\mathrm{Zu}$ vermuten ist diesbezüglich, dass solche Rechtfertigungen das Handlungsziel erfüllen, mögliche politische Allianzen nicht zu gefährden bzw. nicht das eigene Wähler*innenpotenzial zu untergraben.

\subsection{Aufrechnung}

Das Phänomen der aufrechnenden Thematisierung von Antisemitismus findet sich insbesondere im Feld des Sprechens über den nationalsozialistischen Antisemitismus und seine Bedeutung für heute lebende Deutsche. Im Kontrast zur Rechtfertigung wird in aufrechnenden Argumentationen zumindest indirekt anerkannt, dass es sich bei der Vernichtung des europäischen Judentums um ein tatsächliches Unrecht handelt, welches den moralischen Status Deutschlands negativ beeinflusst, und zwar nicht allein in Relation zu Jüd*innen, sondern auch in Relation $\mathrm{zu}$ anderen Nationen. Die damit verbundene moralische Asymmetrie, die zu einem (gefühlten) Ansehensverlust führt, wird sodann aufgelöst, indem der Wir-Gruppe und anderen Gruppen ein »Leid-« oder "Schuldkonto« (vgl. Bergmann 2007: 25, Pollock 1955: 302) zugeschrieben und um Darstellungen ergänzt wird, die Schuld »mathematisch annullier[en]« (vgl. Assmann 2011: 170), um die Konten somit als ausgeglichen erscheinen lassen. Eine verkehrte Form der rhetorischen >Normalisierung ‘ von Außenbeziehungen ist die Folge.

Dies kann rhetorisch auf unterschiedliche Weise realisiert werden. So wird etwa in der Studie zum Gruppenexperiments des Frankfurter Instituts für Sozialforschung auf argumentative Muster hingewiesen, in denen die Schuld aufgrund der psychologischen und materiellen Folgen der deutschen Niederlage im Zweiten Weltkrieg als bereits sgesühnt dargestellt wird (vgl. Pollock 1955: 375). Eine andere Möglichkeit besteht demgegenüber darin, 
$\mathrm{zu}$ argumentieren, dass nicht nur Deutsche, sondern auch andere Nationen Verbrechen begangen hätten (vgl. Giordano 1990: 34). Aleida Assmann präsentiert eine vom Generalgouverneur des nationalsozialistisch besetzten Polens verwendete "Selbstentschuldungsstrategie«, in der die "Schuld von Deutschland « mit den von Russen, Polen und Tschechen vermeintlich begangenen »riesigen Massenverbrechen entsetzlichster Art« verrechnet wird (vgl. Assmann: 170). In einer aktuelleren Studie beobachtet Gudrun Brockhaus ein ähnliches Phänomen, wenn dem Reden über die Verbrechen des Nationalsozialismus durch Verweis auf die von »Türken« und »Amerikanern« begangenen Verbrechen an den Armenier*innen bzw. den Indigenen Nordamerikas begegnet wird (vgl. Brockhaus 2008: 33).

Zuletzt sei darauf hingewiesen, dass sich aufrechnende Argumentationen auch auf Jüd*innen beziehen lassen. Dies funktioniert allerdings nur dann, wenn diesen etwas angedichtet werden kann, was dem Judenmord ähnlich scheint. Sie werden dann als »Tätervolk« präsentiert, ihnen wird eine vermeintliche Beteiligung am »Bolschewismus« oder ihre vermeintliche Verstrickung in den »Weltimperialismus« vorgeworfen (vgl. Bergmann 2007: 28ff.) Von einer NS-vergleichenden Israelkritik kann gesprochen werden, wenn behauptet wird, dass das, was Jüd*innen den Palästinenser*innen vermeintlich antäten, dem entspräche, was ihnen im Nationalsozialismus zugestoßen sei oder als noch schlimmer bewertet werden müsse (vgl. Heyder et al. 2005: 149, Stein 2011: 34). ${ }^{5}$ Entsprechende Aufrechnungen können auch auf indirekte Weise und mit Bezug auf Israel erfolgen, z.B. wenn mithilfe von »Signalwörtern« gearbeitet wird, »die eine eindeutige Nähe [Israels, M. H. ] zur verbrecherischen Politik des nationalsozialistischen Deutschlands suggerieren « (vgl. Betzler/Glittenberg 2015: 40). Im Gedicht »Was gesagt werden muss« findet dies beispielsweise in Bezug auf Israel durch die Verwendung von Wörtern wie »auslöschen«, in Bezug auf Deutsche durch die Bezeichnung »Überlebende« statt (siehe S. 145). 


\subsection{Moralische Diskreditierung}

Bei der moralischen Diskreditierung von vermeintlichen oder tatsächlichen Kritiker*innen des Antisemitismus, von Sykes und Matza als »condemnation of the condemners « (Sykes/Matza 1957: 668) bezeichnet, wird auf die Thematisierung von vergangenem oder gegenwärtigen Antisemitismus reagiert, indem die Antisemitismus Thematisierenden als illegitime Ankläger*innen und damit als eigentliche Täter*innen dargestellt werden. Sie lässt sich damit auch als Gegenmoralisierung verstehen, die eine Auseinandersetzung mit anti-antisemitischer Kritik unnötig erscheinen lässt. Beziehen sich solche ad hominem Vorwürfe auf Jüd*innen, so liegt eine Variante des sekundären Antisemitismus vor (vgl. auch Kapitel 2.9). Diese Form der Abwehr ist schon lange bekannt und stellt eine typische Umgangsweise mit der Erinnerung an Judenverfolgung und Antisemitismus in Deutschland nach 1945 dar. Theodor W. Adorno hat diesbezüglich die sarkastische Formel geprägt, man solle im Hause des Henkers nicht vom Strick reden, sonst gerate man in den Verdacht, man habe Ressentiments (vgl. Adorno 2003: 393).

Entsprechende Rhetoriken zielen laut Potter darauf ab, entgegenstehende Positionen zu unterminieren, indem diese als »talk which is motivated, distorted or erroneous in some way « (Potter 1996: 107) dargestellt werden. Sie können in aktuellen Diskursen über antisemitische Ereignisse auch als Form des abrupten Themenwechsels betrachtet werden, insofern sie die vermeintliche Vorurteiligkeit anderer ins Zentrum der Diskussion stellen. Nach Byford liegt der Vorteil dieser Strategie darin, dass sie die Aufmerksamkeit der Teilnehmer*innen oder Zuschauer*innen eines Konflikts von den mit Antisemitismuskritik verbundenen Problemsetzungen ablenkt (vgl. Byford 2013, IV: 35).

Entsprechende Argumentationen finden sich bereits in klassischen antisemitischen Diskursen. So beklagte beispielsweise Heinrich von Treitschke ein Verfasser von Schlüsseltexten des "nationalen Antisemitismus« (vgl. Holz 2001: 165) - im Jahr 1879 die vermeintliche Stigmatisierung von >Kritikern des Judentums durch Antisemitismuskritik wenn er feststellte:

»Vor wenigen Monaten herrschte in Deutschland noch das berufene >umgekehrte Hep Hep Ceschrei<. Über die Nationalfehler der Deutschen, der Franzosen und aller anderen Völker durfte Jedermann ungescheut das Härteste sagen; wer sich aber unterstand über irgend eine unleugbare Schwäche des jüdischen Charakters gerecht und maßvoll zu reden, ward sofort fast von 
der gesamten Presse als Barbar und Religionsverfolger gebrandmarkt. « (von Treitschke 1879: 8, zitiert nach Holz 2001: 181)

Eine dieser Argumentation ähnliche, vermeintliche `Entlarvungく von anti-antisemitischen Kritiker*innen findet sich auch in frühen antizionistischen Diskursen. So wurde etwa im Zuge eines antizionistischen Schauprozesses in der Sowjetunion der Kommunist Rudolph Slanksy 1952 dazu gezwungen, Folgendes zu erklären:

»I deliberately shielded Zionism by publicly speaking out against the people who pointed to the hostile activities of Zionists and by describing these people as anti-Semites so that these people were in the end prosecuted and persecuted. I thus created an atmosphere in which people were afraid to oppose Zionism.« (Shindler 2011:145f., zitiert nach Hirsh 2013: 8)

David Hirsh merkt an, dass es bei diesen Formen der Argumentation, die er als Livingstone Formulation bezeichnet, häufig ad hominem Vorwürfe im Zentrum der Argumentation stehen, die nicht auf den Inhalt eines anti-antisemitischen Arguments, sondern auf die Diskreditierung derjenigen abzielen, die dieses Argument vertreten:

»the Livingstone Formulation does not simply accuse anyone who raises the issue of contemporary antisemitism of being wrong, it also accuses them of bad faith [...]. Not an honest mistake, but a secret, common plan to try to de-legitimize criticism by means of an instrumental use of a charge of antisemitism.« (Hirsh 2010: 50)

Hirsh problematisiert dies mit dem Hinweis darauf, dass es sich hierbei um eine Form der (diskursiven) Ungerechtigkeit handele, da hierdurch eine Auseinandersetzung mit Argumenten verhindert werde (vgl. ebd.: 48).

Rensmann betont, dass solche Formen der Abwehr oftmals ein Bild des Verhaltens der >Ankläger*innen zeichneten, das mit faktisch getätigten Äußerungen kaum übereinstimmt (Rensmann 2004:345). Formen der Kritik diskreditierenden Abwehr gehen demnach mit Homogenisierungen, voreiligen Generalisierungen und - in den Worten der Argumentationstheorie - mit der Produktion von »Strohmännern« (vgl. Zenker 2008: 14f.) einher, wobei den Proponent"innen bisweilen stärkere Thesen zugeschrieben werden, als diese eigentlich vertreten, oder eine größere Machtstellung zugeschrieben wird, als sie eigentlich haben (vgl. auch Vogt/Vogt 1997: 529, Knothe 2009: 168f.). Sie basiert damit auf verzerrenden Vereindeutigungen, selektiven Lesarten, 
Überstilisierungen usw. In Anschluss an Habermas lassen sich solche Kommunikationsmuster auch als Ausdruck systematisch verzerrter Kommunikation interpretieren. Anstatt hierbei einen »Pseudokonsens « mit antisemitisch Handelnden herzustellen und dadurch einen latenten antisemitischen Konflikt zu neutralisieren, wird hierbei ein Dissens mit Kritiker*innen von Antisemitismus forciert.

Als nachhaltig populär hat sich zudem der mit diskreditierenden Abwehrargumentationen verbundene, generalisierende Topos der >Schuld < bzw. >Kollektivschuld erwiesen. Als Terminus, entstanden in der unmittelbaren Nachkriegszeit (vgl. Kämper 2007: 151), zielte er stets auf die Ablehnung einer aus Auschwitz erwachsenden Schuld und Verantwortung. In der Problematisierung von (Kollektiv-)Schuld wird hierbei die kollektive Identität der Deutschen durch das imaginierte Auge eines Gegenübers betrachtet, deren verurteilender und stigmatisierender Blick die eigene Gruppe als Ganze beträfe. Diese ablehnende Thematisierung von Schuld geht mit der Problematisierung eines vermeintlichen Machtgefälles zwischen Deutschen und Anderen einher: Die lebenden Deutschen seien zwar unschuldig, würden aber illegitimer Weise stigmatisiert (vgl. Vogt/Vogt 1997: 529ff.). Auch für die Problematisierung von (Kollektiv-)Schuld ist die systematische Verzeichnung gesellschaftlicher Realitäten charakteristisch. So wurde und wird ein Kollektivschuldvorwurf von keiner relevanten politischen Gruppe vertreten (vgl. Frei 2005: 145ff., Später 2008). Im Gegensatz zur von Du Bois beschriebenen »Double Consciousness" - der tragischen Notwendigkeit von rassistisch Diskriminierten, sich stets auch durch das Auge der sie umgebenen rassistischen Gesellschaft betrachten zu müssen (vgl. Du Bois 1989:5) - ist das Sprechen von (Kollektiv-)Schuldvorwürfen deshalb als eine Strategie der Selbstviktimisierung von Mitgliedern der Mehrheitsgesellschaft zu bezeichnen. ${ }^{6}$ Diese wird z.B. dann vollzogen, wenn sich die Frage nach den praktischen Konsequenzen der Erinnerung des Antisemitismus stellt oder das Thema Antisemitismus nicht distanziert behandelt werden kann, sondern auch Folgen für das eigene Selbst- und Familienbild hat (vgl. Brockhaus 2008: 32f.). Werner Bergmann zufolge kann dem Sprechen über Schuldvorwürfe deshalb eine rhetorische Funktion zugeschrieben werden, weil es die Möglichkeit bietet, Fragen nach tatsächlicher Schuld und Verantwortung auszuweichen (vgl. Bergmann 2007: 15). Entsprechende Formen der Rede verbinden sich hierbei häufig mit Schlussstrichfor- 
derungen, die bereits unmittelbar nach 1945 und bis in die Gegenwart erhoben werden (vgl. Giordano 1990: 35, Pohl 2010: 234). Die Bedeutung der Aufarbeitung der nationalsozialistischen Vergangenheit wird hierbei meist formal anerkannt und zugleich betont, dass ihre kontinuierliche Fortsetzung als Unrecht zu betrachten sei, dass für die deutsche Wir-Identität mit katastrophalen Folgen oder zumindest unnötigen Kosten verbunden sei.

\subsection{Bagatellisierung}

Unter Bagatellisierungen können Formen abwehrenden Verhaltens verstanden werden, bei denen der antisemitische Charakter einer Handlung oder eines Geschehens zumindest auf indirekte Weise anerkannt wird, um dann allerdings dessen Bedeutung so weit herunterzuspielen, dass es nicht mehr als etwas erscheint, zu dem sich praktisch verhalten werden müsse. Bagatellisierungen gehen also nicht mit Feindbildkonstruktionen einher, sondern zeichnen sich durch ihren defensiven Charakter aus und können sowohl von antisemitisch Handelnden, wie auch ihren Verteidiger*innen als Deutungsangebote in Diskussionen eingebracht werden. Es kommt hierbei zu einer Leugnung der (gesellschaftspolitischen) Relevanz des entsprechenden Ereignisses (vgl. Wetherell/Potter 1992: 212). Mit Rommelspacher lässt sich diesbezüglich darauf verweisen, dass bagatellisierende Argumentationen auch auf Personen bezogen werden können, so etwa, wenn eine der von ihr befragten Kinder von Nationalsozialist*innen in Reaktion auf Antisemitismuskritik beiläufig festgestellt, dass jeder Mensch nun einmal gute und schlechte Seiten in sich trage (vgl. Rommelspacher 1995: 72).

Eine klassische Form der Bagatellisierung der Shoah sind `Zahlenspieleく, wobei etwa behauptet wird, dass gar nicht so viele Jüd*innen umgekommen seien, wie gemeinhin behauptet werde (vgl. Pollock 1955: 292ff., vgl. Giordano 1990: 31). Diese Form der Bagatellisierung ist in öffentlichen Räumen nach deutschem Recht allerdings strafbar. Subtiler sind demgegenüber bagatellisierende Bezeichnungen für die Judenverfolgung. So verweist etwa Adorno auf »mildernde Ausdrücke« oder »euphemistische Beschreibungen«, wodurch sich »ein Hohlraum der Rede« bilde und die industrielle Vernichtung von 6 Millionen Jüd*innen hinter unklaren Bezeichnungen verschwinde (vgl. Pollock 1955: 314, vgl. für weitere Beispiele auch Salzborn 2010: 311f.).

Hinsichtlich des aktuellen Antisemitismus kann bei der bagatellisierenden Abwehr zudem mit Unterscheidungen zwischen relevanten und irrele- 
vanten Formen von Diskriminierung operiert werden (vgl. Verkuyten 1998: 153ff.). Wie im empirischen Teil dieser Arbeit gezeigt werden wird, wird z.B. der Verweis auf >bloße Vorurteile` in Gesprächen über Antisemitismus dafür genutzt, zu begründen, dass diese ein zu vernachlässigendes Problem seien (siehe S. 198f.). Simon Goodman und Lottie Rowe sprechen diesbezüglich von einer >Anerkennung von Vorurteilen, im Dienste ihrer Verleugnung` (vgl. Goodman/Rowe 2014: 43, eigene Übersetzung).

\subsection{Leugnung}

Eine der einfachsten und effektivsten Strategien der Abwehr ist die Leugnung, da mit ihr die Debatte über vergangenen und aktuellen Antisemitismus auf einen Schlag sentsorgt < und - wenn sie erfolgreich ist - der Diskurs über antisemitische Diskriminierung bzw. die Frage, inwiefern solche Diskriminierung Gruppen und Personen zugerechnet werden kann, abrupt beendet wird.

Ein Extremfall der Leugnung von Antisemitismus liegt im Fall der Holocaustleugnung vor (vgl. Lichtenstein 1999, Wetzel 2003, Bergmann 2007: 17ff.). Der rhetorische »Vorteil« dieser Abwehrstrategie liegt offenkundig darin, die mit Judenverfolgung assoziierte Wir-Gruppe der Deutschen und ihrer Mitglieder auf einen Schlag zu rehabilitieren, indem Antisemitismus als Phantasieprodukt ausgewiesen wird. Das Phänomen der Holocaustleugnung korreliert dabei fast zwangsläufig mit auf Jüd*innen bezogenen, sekundärantisemitischen Argumentationen. Meistens wird diesen hierbei zugeschrieben, ein materielles und politisches Interesse daran zu haben, solche Behauptungen zu lancieren. Auch die Holocaustleugnung ist rechtlich sanktioniert und spielt als Abwehrstrategie vor allem für die extreme Rechte eine Rolle (vgl. Benz 2009).

Eine weniger verfängliche Alternative hierzu stellt die Leugnung von Handlungsspielräumen der in die nationalsozialistische Verfolgung involvierten Personen oder die Leugnung eines Wissens um die Verfolgung von Jüd*innen dar. Während im ersten Fall z.B. durch Verweis auf einen vermeintlichen Befehlsnotstand, den es so nie gegeben hat (vgl. Longerich 1998: 310ff.), verbrecherische Praktiken nachträglich gerechtfertigt werden, gewinnt Ralph Giordano zufolge die zweite Abwehrrhetorik ihre Überzeugungskraft dadurch, dass sie um die in den NS-verstrickten Menschen »eine Zone bürgerlicher Gesittung auf[baut], gegen die anzugehen und Widerstand 
zu leisten jegliches Motiv entfiel« (vgl. Giordano 1990: 32). Die Shoah wird hierbei also nicht geleugnet. Doch das Abstreiten eines hierauf bezogenen Wissens ermöglicht es, die Zeitgenoss*innen des Nationalsozialismus von jeglicher Verantwortung freizusprechen.

Allerdings kann sich die Leugnung des Antisemitismus auch auf seine Gegenwart beziehen. Bergmann und Erb verweisen darauf, dass die Existenz eines fortwährenden Antisemitismus bereits in der Nachkriegszeit abgestritten wurde (Bergmann/Erb 1986: 228). Diese Art der Problemverleugnung ist auch heute noch zu beobachten (vgl. Schwarz-Friesel 2015a). Jacqueline Nelson spricht diesbezüglich im Feld der Rassismusforschung von einem »absence discourse «, durch den die Relevanz anti-rassistischer Praxis herabgespielt oder negiert werde (vgl. Nelson 2013: 93, vgl. auch Fozdar 2008: 537). ${ }^{7}$

Van Dijk verweist weiterhin auf Formen der Leugnung, die dann genutzt werden können, wenn Rassismus in Bezug auf konkrete Fälle rassistischer Rede problematisiert wird. Er unterscheidet zwischen vier Möglichkeiten antirassistische Problematisierungen defensiv abzuwehren. Bei Formen des »actdenials« werde geleugnet, dass die inkriminierte Handlung überhaupt stattgefunden habe. Dementsprechend könnte dann sowohl die Verantwortung für die Handlung wie auch für die Handlungsfolgen abgelehnt werden. Die Strategie des »intention-denials« versuche demgegenüber Vorwürfe zu bewältigen, indem reklamiert wird, es bestehe ein Missverständnis bezüglich des gemeinten Inhalts des Gesagten. Weil mit der Äußerung keine bösartigen Absichten verbunden gewesen seien, seien verletzte Gefühle anderer vielleicht verstehbar, aber nicht zu verantworten. ${ }^{8}$ In Strategien des »goal-denials« wird demgegenüber auf die nicht-intendierte diskursiven Resonanz einer Handlung verwiesen. Das Problematisierte habe stattgefunden, aber dass Rassist*innen dem zustimmten, habe nichts mit der getätigten Äußerung zu tun. Beim

Jessica C. Nelson und Kolleg*innen zeigen auf Basis quantitativer Daten, dass Mitglieder der Mehrheitsgesellschaft eher zur Verleugnung von Rassismus tendieren als vom Rassismus betroffene (vgl. Nelson et al. 2013). In Anschluss an Habermas kann diesbezüglich festgehalten werden, dass intention denials deshalb sehr effektiv sind, weil sich - im Unterschied zu Wahrheits-, Richtigkeitsund Verständlichkeitsansprüchen - die Frage, ob jemand wahrhaftig handelt, niemals eindeutig empirisch falsifizieren lässt (vgl. Habermas 2011a: 71). Dementsprechend weist Brianne Hastie bezüglich des Verweises auf Intentionen im Kontext von Rhetoriken der Abwehr darauf hin, dass der Verweis auf »good intentions« häufig als »selfsufficient argument « verwendet werde, d.h. als etwas, das sich von selbst versteht und nicht weiter hinterfragt wird (vgl. Hastie 2009: 713). 
»control denial« werden rassistische Handlungen zum >Ausrutscher< oder >Versehen stilisiert (vgl. van Dijk 1992: 91f.).

Befunde der Antisemitismusforschung können entsprechend dieser Unterscheidungen hier angeschlossen werden. So berichtet etwa Wolfram Stender von Lehrer*innen und Schulsozialarbeiter"innen, die trotz der nachweisbaren Existenz einer Vielzahl von antisemitischen Äußerungen in Klassenzimmern generell bestreiten, dass es solche überhaupt gäbe (vgl. Stender 2011: 243). Weiterhin käme es vor, dass das von Schüler*innen verwendete Schimpfwort >Du Jude! nachträglich in >Du Christ!< umgewandelt werde (ebd.: 244). Es kommt also zu Formen des act-denials. Werner Bergmann verweist demgegenüber darauf, dass sich in antisemitischen Konflikten die Beteiligten durch die »Differenzierung von Motiv und Wirkung« (Bergmann 1997:324) antisemitischer Texte von Vorwürfen des Antisemitismus entlasten könnten. Entsprechende Handlungen lassen sich als Formen des intention-denials bezeichnen. Und Wolfgang Knothe identifiziert in seiner Analyse der Auseinandersetzung zum Umgang mit Antisemitismus bei der globalisierungskritischen NGO Attac unter der Kategorie "Beifall von der falschen Seite « ein Argumentationsschema, dass sich unter dem Begriff des goal-denials subsumieren lässt (vgl. Knothe 2009: 166).

Bei der Leugnung wird bisweilen mit Unterscheidung oder Rekategorisierung gearbeitet, die aber im Gegensatz zur Bagatellisierung nicht mit einer prinzipiellen Anerkennung des problematischen Gehalts einer Äußerung verbunden sind. So verweist etwa Byford auf die Relevanz der Unterscheidung zwischen Antijudaismus und Antisemitismus im serbischen Diskurs, wobei in Reaktion auf Kritik antisemitischer Äußerungen diese als Ausdruck von Antijudaismus rekategorisiert und letzteres als akzeptable ideologische Position ausgewiesen wird (vgl. Byford 2013, V:37ff.). D.h. die Leugnung geht mit einer mit dem Mittel der Rekategorisierung arbeitenden Rechtfertigung einher. Bezüglich der Legitimierung antisemitischer Argumentationen sind demgegenüber die Unterscheidungen zwischen >Antisemitismus und \Israelkritik < bzw. >Antisemitismus und `Antizionismus von großer Bedeutung. Während hierbei formal anerkannt wird, dass eine antisemitische Handlung ein Problem darstellen würde, wird die kritisierte Handlung als legitime Handlung, d.h. demokratische Kritik oder legitime Feindschaft ausgegeben (vgl. SchwarzFriesel 2015b: 303).

Abschließend sei hier auf eine Form der Leugnung von Antisemitismus hingewiesen, die sich nicht direkt auf antisemitische Ereignisse oder Handlungen bezieht, sondern die Möglichkeit eines solchen Ereignisses überhaupt 
negiert. Hierbei kommt es zu einer Imprägnierung der Identität einer Person oder einer Gruppe gegen Antisemitismuskritik. In solchen Fällen wird ein »quasi-syllogistischer Schluss« (Schwarz-Friesel 2012: 354, vgl. auch Stein 2011: 73) vorgenommen, der auf der Oberprämisse beruht, dass Menschen z.B. aufgrund politischer Orientierung oder besonderer Leistungen nicht antisemitisch handeln könnten. ${ }^{9}$ Unter entsprechenden Leugnungen sind u.a. solche Argumentationen zu fassen, bei denen unter Verweis darauf, dass sich Antifaschismus und Antisemitismus ausschlössen, abgestritten wird, dass es das Phänomen eines linken Antisemitismus überhaupt geben könnte. Ein analoges Argument besteht darin, zu behaupten, dass Araber*innen nicht antisemitisch eingestellt sein können, da sie selbst >Semiten seien (vgl. Bergmann 2006, Knothe 2015: 27f., Arnold 2016: 286). ${ }^{10}$

\subsection{Externalisierung und Extremisierung von Antisemitismus}

Die Portraitierung verschiedener Formen von Abwehrhandlungen soll nun durch die Beschreibung von zwei Deutungsmustern abgeschlossen werden, die nicht als eigenständige Abwehrstrategien beschrieben werden können, insofern sie logisch im Kontext anderer Abwehrhandlungen zu verorten sind und ihrer Plausibilisierung dort dienen, wo die inhaltliche Schwäche einer Abwehrargumentation ihre Zurückweisung wahrscheinlich macht. Sie fungieren hierbei gewissermaßen als argumentatives Schmiermittel, das zunächst von potenziellen Antisemitismuskritiker*innen effektiv infrage gestellt werden müsste, um gegen die eigentliche Abwehrstrategie überhaupt angehen zu können. Erschwert wird dies allerdings durch die Verankerung dieser Deutungsmuster im Bestand des als selbstverständlich geltenden Alltagswissens.

Hierzu gehört zum einen die Externalisierung von Antisemitismus. Dieses Deutungsmuster wird Byford zufolge vor allem deshalb im Kontext von

Bergmann diskutiert das Beispiel einer Debatte im Jahr 1967 über antisemitische Äußerungen des Kardinals Joseph Frings. Hier hätten Vertreterinnen der Medien nicht versucht »den Fall zu skandalisieren, sondern [...] Vorwürfe mit Entlastungsargumenten aus[balanciert] «da Frings eine hohe kirchliche Position innehatte und »als Gegner des Nationalsozialismus bekannt war, was für viele offenbar die Möglichkeit antisemitischer Einstellungen ausschloß« (Bergmann 1997: 294f.).

10 Siehe hierzu auch die Ausführungen zum demokratischen Antisemitismus in Kapitel 2.1, der im Unterschied hierzu darauf abzielt, dass die Rezipient"innen einer antisemitischen Äußerung, den quasi-syllogistischen Schluss gedanklich selbst vollziehen. 
Abwehrhandlungen verwendet, weil diese größere Überzeugungskraft gewinnen "when at least some form of deviation from the ideal is admitted to (Byford 2013, IV: 55). Antisemitismus wird hierbei also durchaus als Problem anerkannt, um es zugleich aber so zu verorten, dass es nicht mit der Wir-Gruppe oder der eigenen Person in Verbindung gebracht werden kann. Das Problem erscheint damit als »Antisemitismus der anderen« (Ullich 2014: 117) und nicht als eigenes Problem. Diesbezüglich kann zwischen mehreren Varianten unterschieden werden. Bei Nelson werden unter der stemporal deflection« solche Argumentationen gefasst, die Rassismus als Problem der Vergangenheit abtun. Unter der "spatial deflection « werden demgegenüber Problematisierungen gefasst, die Rassismus allein in anderen Ländern, Regionen oder Gruppen verortet. Als »deflection from the mainstream « werden Externalisierungen verstanden, die Rassismus an den Rändern der Gesellschaft verorten (vgl. Nelson 2013: 93). Gemein haben diese Formen, dass sie die Vorstellung einer Verbreitung von Vorurteilen und Diskriminierung in der Mitte der Gesellschaft weitgehend abstreiten. Bisherige Befunde der Antisemitismusforschung lassen sich sehr gut unter diese Begriffe subsumieren. So wird beispielsweise Antisemitismus in Serbien vor allem als von anderen Ländern importiertes Problem behandelt und somit räumlich externalisiert (vgl. Byford 2013, IV: 41). Hier$\mathrm{zu}$ kommt es aktuell auch in deutschen Diskursen, wenn Antisemitismus als durch Flüchtlinge importiertes Problem dargestellt wird (vgl. Amadeu Antonio Stiftung 2017: 8). Historisch betrachtet werden in Deutschland allerdings vor allem Formen sozialer Externalisierung innerhalb der deutschen Bezugsgruppe tradiert. Hierzu gehört z.B. der Mythos, dass der nationalsozialistische Antisemitismus nur eine kleine Gruppe um Hitler betraf. Dieser war nicht nur in der Nachkriegszeit äußerst populär (vgl. Möller 2006), sondern blieb auf der Ebene von Familienerzählungen immer relevant (vgl. Welzer et al. 2002: 81ff.). Shida Kiani kommt in seiner Analyse der Berichterstattung über die antisemitische Schmierwelle von $1959 \mathrm{zu}$ dem Ergebnis, dass hier Antisemitismus insbesondere den »Halbstarken« zugeschrieben wurde (vgl. Kiani 2008: 127ff.). Ljiljana Radonić berichtet davon, dass in der Frauenbewegung Antisemitismus als Problem von Männern bzw. Männerbünden dargestellt wurde (Radonić 2016: 204ff., vgl. auch Eschebach/Wenk 2002: 26). Timo Stein stellt fest, dass in gewissen Spektren der politischen Linken insbesondere eine Externalisierung von Antisemitismus auf den Faschismus stattfindet (vgl. Stein: 74f.). Und Guido Follert und Wolfram Stender problematisieren in ihrer Analyse der Thematisierung von Antisemitismus durch nicht-jüdische deutsche Lehrer*innen die gängige Auffassung, dass Antisemitismus nur un- 
ter Muslim*innen verbreitet sei (Follert/Stender 2010). Auch sind Formen der temporalen Externalisierung von Antisemitismus nach wie vor relevant. Antisemitismus wird hierbei als Problem der Vergangenheit abgetan und auf die Judenvernichtung reduziert (vgl. Schwarz-Friesel 2015b: 23f.).

Der rhetorische Vorteil von Formen dieses »Othering« des Antisemitismus (vgl. Stender 2008: 7) liegt darin, dass durch sie die eigene moralische Integrität im Zusammenhang mit Abwehrhandlungen zum Ausdruck gebracht werden kann, um dies auf eine Weise zu tun, die kein schlechtes Licht auf die eigene Gruppe oder das eigene Land wirft. Diese diskursive Konstruktion von Fremdheit findet allerdings nicht allein hinsichtlich der Gruppe der >Antisemiten<, sondern auch hinsichtlich des Konzepts >Antisemitismus< selbst statt. So weisen etwa Kate L. Harris und Kolleg*innen auf den Umstand hin, dass die extremisierende Darstellung von Rassismus zu einem funktionalen Bestandteil von Abwehrrhetoriken gemacht werden könne:

"[I]n order to defend against criticism, excuse a person of responsibility, and justify behaviors, people will sometimes use extreme cases [...]. By emphasizing the exceptional qualities of some thought, belief, or action, those who converse determine appropriate actions through comparison« (Harris et al. 2012: 650).

Byford macht in seiner Analyse der Extremisierung von Antisemitismus in Serbien eine ähnliche Beobachtung. Antisemitische Verhaltensweisen würden hierbei verleugnet, indem eine "favorable comparison with a more radical view that is unambiguously extremist « (Byford 2013, V: 46) vorgenommen werde (vgl. auch Billig 1988: 104). Diese Beobachtung passt zu der Feststellung Maykel Verkuytens, dass bei Formen der Abwehr von Rassismuskritik häufig eine Unterscheidung zwischen sechtem Rassismus, der insbesondere mit der Ermordung der europäischen Jüd*innen aussoziiert wird, und nicht-relevanten >Vorurteilen`vorgenommen werde. Die Shoah diene hierbei als Kontrastfolie, um die eigene Meinung zu legitimieren und um vermeintlich $\mathrm{zu}$ beweisen, dass man selbst nicht rassistisch sei (vgl. auch Verkuyten 1998: 153). Die Extremisierung von Antisemitismus wird hierbei also in Strategien der Leugnung eingebunden.

Von Vertreter*innen der diskursiven Psychologie ist darauf hingewiesen worden, dass Rassismus in öffentlichen Diskursen häufig als »aberration« (Riggs/Due 2010: 262) dargestellt wird und >die Rassisten als irrational gelten (vgl. auch Goodman/Rowe 2014: 33). Diese Vorstellungen dokumentieren sich auch in Antisemitismusdiskursen. So hat die Linguistin und 
Antisemitismusforscherin Monika Schwarz-Friesel festgestellt, dass das "moderne Post-Holocaust-Bewusstsein [...] Judenfeindschaft als etwas grotesk Inhumanes und Wahnhaftes « (Schwarz-Friesel 2015b: 307; vgl. auch Betzler/Glittenberg: 209f.) betrachte. Schwarz-Friesel stellt diesbezüglich allerdings eine These auf, die den Überlegungen aus dem Feld der diskursiven Psychologie widerspricht. Die Autorin geht nicht davon aus, dass die Extremisierung als Bestandteil von Abwehrrhetoriken, sondern umgekehrt, dass die Nicht-Anerkennung der Gegenwart des Antisemitismus als Resultat von extremisierenden Vorstellungskomplexen $\mathrm{zu}$ verstehen sei (SchwarzFriesel 2015a: 14). Dieser Widerspruch lässt sich entweder ausbauen oder theoretisch auflösen. Er lässt sich ausbauen, wenn Schwarz-Friesels These um eine Kritik gesellschaftlicher Formen der Wissensvermittlung von Antisemitismus erweitert wird. So lässt sich mit Michael Kohlstruck und Peter Ullrich sagen, dass in öffentlichen anti-antisemitischen Diskursen das Wort >Antisemitismus« oftmals als "generalisiertes negatives politischen Symbol« (Kohstruck/Ullrich 2014: 11) fungiert, das Antisemitismus auf die Dimension der Ermordung und Vernichtung von Jüd*innen reduziert. Vor diesem Hintergrund ist davon auszugehen, dass hierdurch auch ein reduktionistisches Alltagsverständnis von Antisemitismus geprägt wird. ${ }^{11}$ Demgegenüber lässt sich der Widerspruch auflösen, wenn an die in Kapitel 1.6 entwickelte Überlegung angeschlossen wird, dass sich die Abwehr von Antisemitismus als kulturprägend erweist. In diesem Fall scheint es angemessen, von einem zirkulären Prozess auszugehen, wobei die Extremisierung von Antisemitismus zugleich die strategische Funktion erfüllt, Antisemitismusvorwürfe als »überzogen« zurückzuweisen, wie solche Handlungen umgekehrt auch alltägliche Vorstellungen davon, was Antisemitismus sei, prägen.

11 Kritisch ist hierzu anzumerken, dass die Autoren in ihrer Studie zu anti-antisemitischen Interventionen in Berliner (Bildungs-)Institutionen diese Überlegung theoretisch so kontextualisieren, dass weder die mögliche Abwehrfunktion dieses Reduktionismus, noch die Differenziertheit öffentlicher Antisemitismuskritik oder die durch Abwehrphänomene begrenzte Wirkungsweise anti-antisemischer Interventionen überhaupt noch in den Blick der eigenen Analyse geraten können (vgl. ebd.: 2off.). 


\subsection{Sekundärer Antisemitismus}

Die Übersicht über die verschiedenen Formen der Abwehr der Thematisierung und Kritik von Antisemitismus wird nun mit einem Exkurs zum sekundären Antisemitismus abgeschlossen. ${ }^{12}$ Vor dem Hintergrund der bisherigen Befunde ist der sekundäre Antisemitismus sowohl als psychologisches wie auch soziales Abwehrphänomen zu verstehen. Aus rhetorischer Perspektive handelt es sich beim sekundären Antisemitismus nicht um eine eindeutig abgrenzbare Abwehrhandlung, sondern um eine Kombination verschiedener Rhetoriken der Abwehr und ihre antisemitische Zuspitzung oder Codierung. Dem sekundären Antisemitismus unterliegen hierbei latente Handlungsmotive, die im Theorieteil der Arbeit bereits an verschiedenen Stellen benannt wurden und auf die hier noch einmal näher eingegangen wird.

Sekundär heißt bildungssprachlich snach einem bestimmten Ereignis kommend . In diesem Sinne stellt der sekundäre Antisemitismus eine Familie von Antisemitismen dar, die sich: 1. nach Auschwitz aktualisiert und an neue gesellschaftliche Bedingungen angepasst haben, 2 . darauf abzielen, die politischen und kulturellen Folgen der Aufarbeitung des nationalsozialistischen Judenmords (insbesondere die Entstehung einer anti-antisemitischen Öffentlichkeit) auf antisemitische Weise zu neutralisieren sowie 3. dazu dienen, psychologische Ambivalenzen zu kaschieren, die aus einer gescheiterten individuellen Aufarbeitung der NS-Vergangenheit resultieren.

Ad 1) Zunächst sind alle Modifikationen des Antisemitismus nach Auschwitz (siehe auch Kapitel 2.1) als Ausdruck des sekundären Antisemitismus zu verstehen. Um Abwehrhandlungen handelt es sich hierbei insofern, als sie darauf abzielen, Antisemitismus zum Ausdruck zu bringen, ohne sich Kritik und gesellschaftliche Sanktionen aussetzen zu müssen.

Ad 2) In einer frühen Antisemitismusstudie des Frankfurter Instituts für Sozialforschung stellte Peter Schönbach die Überlegung auf, dass die in den Nationalsozialismus involvierte erste Generation auf den öffentlichen Anti-Antisemitismus reagierte, indem sie sich innerhalb des Familienzusammenhangs für ihre Verstricktheit durch nachträgliche Rechtfertigungen der Judenverfolgung (siehe Kapitel 2.3) entschuldigte (vgl. Schönbach 1961:

12 Dieses Kapitel basiert auf dem ursprünglich von der Bundeszentrale für politische Bildung/bpb herausgegebenen Text »Sekundärer Antisemitismus « im Online Dossier zu Antisemitismus (vgl. Höttemann 2020). 
80). Rechtfertigungen von Antisemitismus dienen hierbei der Stabilisierung von sozialen Beziehungen, insofern als den Opfern zumindest eine Mitschuld an ihrer Verfolgung zugeschrieben wird. Hieran können dann Prozesse der innerfamiliären Tradierung des Antisemitismus anschließen zumindest dann, wenn die zweite Generation die antisemitischen Einstellungen der Eltern übernimmt, um die für sie identitätsstiftenden Bilder von Vater und Mutter moralisch rein zu halten (siehe Kapitel 1.7 zur Abwehr in Familienkonstellationen). ${ }^{13}$

Variationen sekundärantisemitischer Gegenreaktionen auf Antisemitismuskritik lassen sich auch in anderen Sozialzusammenhängen beobachten. Sie dienen stets dazu, sich selbst oder unter Druck geratene Sozialzusammenhänge (z.B. von Antisemitismuskritik betroffene Parteien) sowie sozial oder politisch nahestehende Personen gegen Antisemitismuskritik abzuschirmen. Neben dem von Schönbach beschriebenen Rechtfertigungsreaktionen kommt es hierbei auch dazu, dass antisemitismuskritische Positionen auf antisemitische Weise moralischen diskreditiert werden (siehe auch Kapitel 2.5). Jüd*innen oder mit Jüd*innen assoziierte Institutionen (z.B. der Zentralrat der Juden in Deutschland oder Israel) werden hierbei durch die Verwendung von elaborierten Argumentationen oder Stereotypen zu unfairen, geldgierigen oder rachsüchtigen »Störenfrieden der Erinnerung (Bergmann 2007) stilisiert. Typisch ist auch den Opfern eine Befangenheit bzgl. des Themas Antisemitismus zu unterstellen, d.h. zu behaupten, dass diese nicht dazu in der Lage seien, >objektiv< über Antisemitismus zu sprechen.

Dem sekundären Antisemitismus kommt ebenfalls im Zusammengang mit Nationalismuskritik und dem damit verbundenen Versuch die kollektive Identität vom >Stigma Antisemitismus zu befreien eine Rolle zu. Als »Paradoxie der Normalisierung« (Holz 2007) wird der Umstand bezeichnet, dass die Etablierung einer anti-antisemitischen Erinnerungskultur den deutschen Nationalismus nach 1945 in eine widersprüchliche Lage gebracht hat. Einerseits ist die positive Bezugnahme auf die Idee einer deutschen Nation aus 
dem Feld der Politik nicht einfach verschwunden. Andererseits hat sich in zunehmendem Maße ein politisches Paradigma etabliert, wonach sich der positive Bezug auf die Nation nur durch die Abgrenzung von den Verbrechen der Nationalsozialisten legitimieren lässt. Dies aber wiederum erschwert eine restlose Normalisierung deutscher Identität, und verunmöglicht sie dort, wo das Bedürfnis nach einer bruchlosen Identifikation mit der deutschen Geschichte sich Bahn bricht. Wer der begrenzten politischen Identifikationsmöglichkeit den Stachel ziehen will, muss die Bedeutung der Erinnerung (durch Bagatellisierung oder Aufrechnung) relativieren bzw. diskreditieren oder die in der Paradoxie der Normalisierung enthaltene Nationalismuskritik selber angreifen. Heute spielen insbesondere rechtsextreme Politiker(innen) auf der Klaviatur sekundär-antisemitischer Rhetoriken. Die Wirkmacht von nationalismuskritischen Normen wird hierbei direkt auf die vermeintlichen Macht >der Juden $<$ zurückgeführt. Dies ist z B. dann der Fall, wenn behauptet wird, Jüd*innen würden Macht über die Deutschen ausüben, z.B. indem sie eine »Erziehungsdiktatur« (Benz 2004: 49) errichtet hätten.

Ad 3) Sekundärer Antisemitismus zur Neutralisierung von Nationalismuskritik hat nicht nur eine politische, sondern auch eine psychologische Abwehrdimension, deren Bedeutung in Abschnitt zu der psychoanalytischen Grundlegung des Abwehrbegriffs bereits diskutiert wurde (siehe Kapitel 1.1). In entsprechenden Erklärungsansätzen wird vorausgesetzt, dass die »überwertige Identifikation« (vgl. Pollock 1955: 281) mit der deutschen Nation durch ein zumindest latentes Bewusstsein über die Bedeutung von Ausschwitz zugleich auch infrage gestellt wird. Die daraus resultierende Frustration wird bewältigt, indem der Widerspruch als Ausdruck einer »die Deutschen « stigmatisierende Erinnerungskultur imaginiert und ihre Entstehung auf jüdischen Einfluss zurückgeführt wird. Mit der Vorstellung, ein Opfer einer auf diese Weise dominierten Erinnerungskultur zu sein, lässt es sich dann leichter leben, als mit der Erkenntnis, sich im Grunde nicht mit der nationalsozialistischen Vergangenheit auseinandersetzen $\mathrm{zu}$ wollen. Es ist deshalb kaum verwunderlich, dass der sekundäre Antisemitismus häufig in Situationen auftaucht, in denen die Frage nach der historischen Verantwortung heute lebender Deutscher relevant wird oder die Frage diskutiert wird, wie heute an die im Nationalsozialismus begangenen Verbrechen erinnert werden sollte.

Eine weitere psychologische Erklärung des sekundären Antisemitismus basiert auf der in der Antisemitismusforschung einschlägigen Überlegung, dass in der Kriegsgeneration Schuld- und Schamgefühle zumindest latent 
vorhanden waren und aus den Verstrickungen in das nationalsozialistische Verfolgungssystem resultieren. Im sekundären Antisemitismus werden diese unbequemen Gefühle durch den psychologischen Mechanismus der Projektion abgewehrt. Schuld und Schamgefühlen wird ausgewichen, indem Jüdinnen und Juden sowie jüdische Institutionen als rachsüchtig und nachtragend imaginiert werden. Der israelische Psychoanalytiker Zwi Rix soll diese Überlegung einmal durch die paradox anmutende Formulierung ausgedrückt haben, dass die Deutschen den Juden Auschwitz niemals verzeihen werden (vgl. Heinsohn 1988: 115). Manche Autor*innen gehen davon aus, dass Schuldgefühle von den nachfolgenden Generationen übernommen werden, wenn diese nicht aufgearbeitet worden seien. Dann ist von einer transgenerationellen Weitergabe von Schuld bzw. von »entlehnten Schuldgefühlen « (Vogt/Vogt 1997: 500ff.) die Rede. Deren Spezifikum bestehe darin, dass sie keinen wirklichen Inhalt hätten, da die Frage nach der Schuld »aus ihrem Zusammenhang genommen und dadurch unkenntlich gemacht « (Rommelspacher 1995: 96) worden sei. Anstatt den Ursachen dieser Emotionen auf den Grund zu gehen, was die Nachgeborenen auch in Konfliktstellung zu ihren Eltern und Großeltern bringen würde, werde die »Ursache ihrer eigenen Schuldgefühle ungebremst auf die Juden [projiziert], die anscheinend immer Schuldabgleichung fordern, anstatt sie in der Beziehung zu ihren Eltern zu suchen« (ebd.: 125). In diesem Zusammenhang können dann auch klassische antisemitische Stereotype - z.B. »Rachsucht« und »Geldgier« im Zusammenhang von Entschädigungsforderungen - aktualisiert werden. Auch kann bereits die Begegnung mit Jüdinnen und Juden entlehnte Schuldgefühle und als Reaktion darauf sekundärantisemitische Abwehrreaktionen auslösen.

Die Tatsache, dass verschiedene Erklärungsansätze für sekundärantisemitische Phänomene vorliegen, lässt sich als Ausdruck der Komplexität dieses Abwehrphänomens verstehen. Sekundärer Antisemitismus verweist zugleich auf eine antisemitische Deutung des Anti-Antisemitismus nach Auschwitz, den Versuch diesen zu unterlaufen und zu delegitimieren und - im psychologischen Sinn - auch auf dessen Scheitern.

\subsection{Zwischenfazit}

Im nun abzuschließenden Kapitel wurde deutlich, dass sich Abwehrhandlungen zwar durch ihre Vielfalt auszeichnen, sich allerdings in dieser Vielfalt auch unter diskrete Kategorien subsumieren lassen (siehe Abbildung S. 113). 
Dies ist an sich bereits ein wichtiger Befund, zeigt er doch, dass es sich bei Abwehrhandlungen offenbar um regelgeleitete soziale Praktiken handelt und es - abgesehen von der Kategorie der Modifikation antisemitischer Rede bezüglich der Oberkategorien auch keinen prinzipiellen Unterschied macht, ob sich abwehrendes Verhalten auf die Thematisierung von vergangenem oder gegenwärtigem Antisemitismus bezieht. Diese Vielfalt erklärt sich vor allem durch die Variationsbreite innerhalb der Kategorien bzw. dadurch, dass Abwehrhandlungen in verschiedenen sozialen Kontexten funktional werden können. So macht es beispielsweise einen Unterschied, ob Abwehrhandlungen proaktiv oder reaktiv erfolgen. Kritik antizipierende Abwehrhandlungen zielen darauf, einen möglichen Antisemitismuskonflikt gar nicht erst entstehen zu lassen. Reaktive Formen können demgegenüber auftreten, wenn sich ein Konflikt nicht mehr vermeiden lässt oder sich eine Kultur der Kritik des Antisemitismus oder eine Erinnerungskultur etabliert hat. Weiterhin ist es bedeutsam, ob Abwehr auf eine Verteidigung von Gruppenidentitäten oder die Stabilisierung einer positiven Identität von Individuen zielt. Die reaktive Abwehr rangiert zudem zwischen defensiven und offensiven Ausdrucksformen. Eine defensive Ausrichtung der Rede zielt u.a. darauf, die von Antisemitismuskritik betroffene Individuen oder Gruppen durch positive Beschreibungen vom möglichen Verdacht des Vorurteils zu befreien oder das Verhalten als entschuldbar darzustellen. Offensive Formen rechtfertigen demgegenüber antisemitische Handlungen oder gehen zu einem Angriff auf die Kritiker*innen über. Mit den Soziologen Peter Luckmann und Thomas Berger kann diesbezüglich angenommen werden, dass »die Härte solcher Verteidigungsmaßnahmen [...] in Proportion zur mutmaßlichen Ernsthaftigkeit der Bedrohung« steht, allerdings auch zur Routine werden kann, um mit Gegenpositionen umzugehen (vgl. Luckmann/Berger 1980: 166). ${ }^{14}$

Des Weiteren lässt sich zwischen solchen Formen der Abwehr unterscheiden, die auf spezifische Handlungen und Situationen bezogen bleibt oder die weltanschaulich entfaltet, und das heißt gesellschaftlich und historisch kontextualisiert werden, wie dies z.B. beim sekundären Antisemitismus und beim Sprechen über eine vermeintliche Kollektivschuld und bei Deckererinnerung der Fall ist. Zuletzt lässt sich zwischen solchen Formen der Abwehr

14 Die Autoren beziehen diese Überlegungen auf den Umgang mit Krisensituationen, in denen Akteuren*innen sihre`subjektive Wirklichkeit - inklusiver aller der mit ihr verbundenen Cewissheiten und Garantien - abhanden zu kommen droht (vgl. ebd.). 
unterscheiden, die Antisemitismus zumindest partiell als Problem anerkennen und solchen, die das nicht tun. Gerade bei Letzteren ist es wahrscheinlich, dass hierbei auch bestimmte Facetten antisemitischer Stereotypie reproduziert werden. Insbesondere beim sekundären Antisemitismus wird deutlich, dass dieser nicht prinzipiell vom Primären unterschieden werden kann, da letzterer auch im Gewand des ersten reproduziert wird. Letztendlich arbeiten beide unter historisch differenten Bedingungen an der Stilisierung von Tätern zu Opfern, und jüdischen Opfern zu Tätern, um das Verhältnis beider zueinander unter Verwendung typischer antisemitischer Argumentationsmuster und Semantiken zu bestimmen.

Zudem ist zu betonen, dass Abwehrhandlungen meist nicht isoliert voneinander auftreten, sondern dass diese sich in gewissem Graden auch miteinander kombinieren lassen. So verweist David Hirsch unter der Bezeichnung "Livingstone Rhetoric « auf die Kombination von Antisemitismus leugnenden Formen der Abwehr mit Thematisierungsverweigerungen und der moralischen Diskreditierung von Antisemitismuskritiker*innen (vgl. Hirsh 2010). Wird diese Form der Rhetorik antisemitisch codiert handelt es sich um eine Variante des sekundären Antisemitismus.

Abschließend ist noch zu fragen, inwiefern die porträtierten Formen der Abwehr dazu geeignet sind, aufgrund ihrer kulturellen Tradierung (in öffentlichen Diskursen, in Familien, Organisationen usw.), gesellschaftlich verbreitete Konsensstrukturen und letztlich auch das Alltagswissens zu prägen und somit dazu führen, dass auch Individuen für die Abwehrhandlungen keine Funktion erfüllt auf die Thematisierung von Antisemitismus aus wertrationalen Erwägungen heraus mit Ablehnung reagieren. Theoretisch betrachtet eignen sich hierfür alle Formen argumentativ strukturierter Abwehrrhetoriken, sowie die Handlung des Beschweigens. In Anschluss an die oben entwickelten theoretischen Überlegungen können diesbezüglich verschiedene Hypothesen aufgestellt werden:

- Formen des Schweigens, sowie der Leugnung und Bagatellisierung von Antisemitismus führen im Fall ihrer Tradierung zur Prägung von Überzeugungen über die Verbreitung von Antisemitismus in Vergangenheit und Gegenwart. Erweisen sich solche Verhaltensweisen wiederholt als erfolgreich, so prägt dies die Vorstellung, dass Antisemitismus kein oder kein relevantes Problem bestimmter Gruppen oder Gesellschaften war oder ist. 
- Die Tradierung von Rechtfertigungen und Innovationen des Antisemitismus sind dazu geeignet, Eingang in den gesellschaftlich tradierten Wissensbestand über vermeintliche Eigenschaften von Jüd*innen $\mathrm{zu}$ finden. D.h. sie können insbesondere zur Etablierung antisemitischer Konsensmuster wie den demokratischen und israelbezogenen Antisemitismus, des sekundären Antisemitismus und der antisemitischen Separation (Jüd*innen als illoyale Fremde) führen.

- Demgegenüber scheint die Normalisierung der moralischen Diskreditierung sowohl dazu geeignet zu sein, Vorstellungen über die Natur von Thematisierungen des Antisemitismus als instrumentell, politisch motiviert oder bösartig zu prägen, zugleich aber auch die Vorstellung zu bestimmen, dass es unmöglich sei, demokratische Kritik an Jüd*innen und Israel offen zu äußern. Dies hat kann zum einen zur Folge haben, dass Antisemitisches tatsächlich sagbar wird. Wie auch die Aufrechnung dürfte sie zudem bewirken, dass sich die durch Fakten nicht gedeckte Idee etabliert, dass Deutsche in der Welt immer noch als Schuldige betrachtet werden.

- Die mit Abwehr einhergehenden Plausibilisierungsmuster der Extremisierung und Externalisierung können demgegenüber laientheoretische Vorstellungen über die Natur von Antisemitismus als exzeptionell, als Problem anderer Gruppen, Nationen und Zeiten inhaltlich (vor-)strukturieren.

Diesbezüglich ist abschließend zu betonen, dass es sich bei diesen Überlegungen zwar um theoretisch plausible Argumente handelt, ein empirischer Nachweis über diese Prozesse bzw. ihre Relevanz als Erklärungsansatz an dieser Stelle allerdings nicht vorgenommen werden kann. Denn hierfür fehlen entsprechende Vergleichsstudien mit einem ähnlichen theoretischen Rahmen. Im gegebenen Kontext dient der Verweis auf diese Phänomene deshalb vor allem dazu, auf die nicht vermeidbaren Risiken zu verweisen, die damit einhergehen, Formen der Argumentation, die Abwehrargumentationen reproduzieren, unmittelbar als funktionale Rhetoriken auszuweisen, die für Individuen bestimmte Funktionen übernehmen. 
Abbildung 3: Überblick über Abwehrhandlungen und ihre Subtypen

\begin{tabular}{|c|c|c|}
\hline & Abwehrhandlung & Subtypen \\
\hline & $\begin{array}{l}\text { Modifikation } \\
\text { antisemitischer } \\
\text { Äußerungen }\end{array}$ & $\begin{array}{l}\text { - Modifikation antisemitischer Stereotype } \\
\text { (Sekundärer Antisemitismus) } \\
\text { - Modifizierte Einführung antisemitischer Stereotype } \\
\text { - Modifizierung des Äußerungsmodus von } \\
\text { Antisemitismus } \\
\text { - Umwegkommunikation }\end{array}$ \\
\hline & Aufrechnung & $\begin{array}{l}\text { - Aufrechnung ohne die Reproduktion } \\
\text { antisemitischer Stereotype } \\
\text { - Aufrechnung durch die Reproduktion antisemiti- } \\
\text { scher Stereotype (Sekundärer Antisemitismus) }\end{array}$ \\
\hline & Rechtfertigung & $\begin{array}{l}\text { - volle Rechtfertigung (Sekundärer Antisemitismus) } \\
\text { - abgeschwächte Rechtfertigung } \\
\text { - Behauptung einer Katalysatorfunktion des } \\
\text { Antisemitismus (Rechtfertigung >positiver } \\
\text { Wirkungen antisemitischer Ideologie) }\end{array}$ \\
\hline & $\begin{array}{l}\text { Kommunikations- } \\
\text { vermeidung }\end{array}$ & $\begin{array}{l}\text { - Nicht-Kommunikation antisemitischer } \\
\text { Einstellungen } \\
\text { - vom Thema Antisemitismus ablenkende Rede } \\
\text { - forcierte Themenwechsel } \\
\text { - Ersatzmythen } \\
\text { - (Be-)schweigen (bestimmter Formen von } \\
\text { Antisemitismus) }\end{array}$ \\
\hline \multirow{3}{*}{ 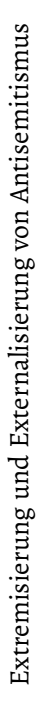 } & $\begin{array}{l}\text { Moralische } \\
\text { Diskreditierung }\end{array}$ & $\begin{array}{l}\text { - moralische Diskreditierung ohne die Reproduktion } \\
\text { antisemitischer Stereotype } \\
\text { - Diskreditierung durch die Reproduktion antisemiti- } \\
\text { scher Stereotype (Sekundärer Antisemitismus) }\end{array}$ \\
\hline & Bagatellisierung & $\begin{array}{l}\text { - Relativierungen und Euphemismen } \\
\text { - bagatellisierende Unterscheidungen }\end{array}$ \\
\hline & Leugnung & $\begin{array}{l}\text { - Leugnung des antisemitischen Ereignisses oder } \\
\text { Phänomens } \\
\text { - Leugnung des Wissens um das Ereignis (Shoah) } \\
\text { - Leugnung von Handlungsspielräumen (Shoah) } \\
\text { - Intention-Denial (Antisemitisches nicht } \\
\text { so gemeint) } \\
\text { - Goal-Denial (antisemitische Resonanz nicht } \\
\text { intendiert) } \\
\text { - Control-Denial (versehentlicher Antisemitismus) } \\
\text { - Immunisierung individueller, sozialer, politischer } \\
\text { und kultureller Identitäten gegen Antisemitismus- } \\
\text { kritik }\end{array}$ \\
\hline
\end{tabular}


